with acute pancreatitis and lupus nephritis was considered.Further investigations were suggestive of lupus(table). Workup for APLA revealed positive lupus anticoagulant.Her skin biopsy was consistent with lupus and renal biopsy revealed class 4 lupus nephritis.She was initiated on oral prednisolone and was given pulses of intravenous cyclophosphamide.There has been no recurrence of pancreatitis over 12 years follow-up.

Conclusions Pancreatitis can at times, be the presentation of childhood lupus and requires prompt and aggressive management.

Funding Source(s): None

\section{STIMULATION OF MONONUCLEAR CELLS THROUGH TOLL-LIKE RECEPTOR 9 INDUCES RELEASE OF MICROVESICLES EXPRESSING DNA AND GALECTIN 3- BINDING PROTEIN IN AN INTERFERON-DEPENDENT MANNER}

${ }^{1}$ Soren Jacobsen*, ${ }^{1}$ Niclas Stefan Rasmussen, ${ }^{1}$ Christoffer Tandrup Nielsen, ${ }^{2}$ Claus Henrik Nielsen. 'Lupus and Vasculitis Clinic, Rheumatology, Copenhagen University Hospital, Rigshospitalet; ${ }^{2}$ Institute of Inflammation Research, Rheumatology, Copenhagen University Hospital, Rigshospitalet

10.1136/lupus-2019-Ism.131
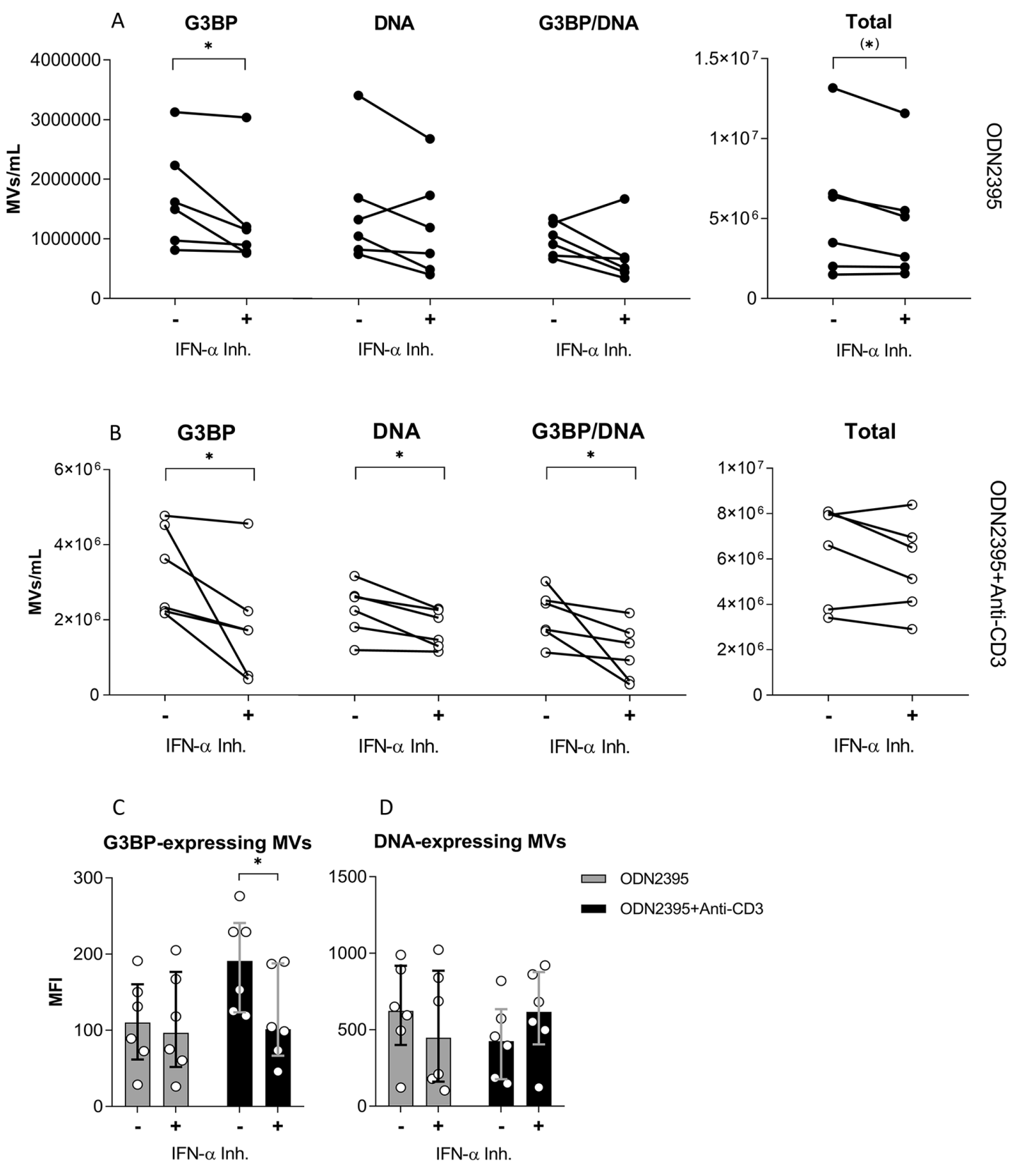

D

DNA-expressing MVs

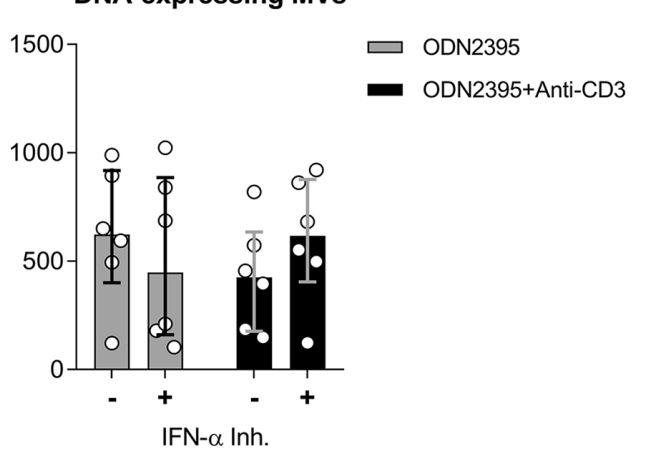

Abstract 131 Figure 1 PBMCs from healthy donors $(n=6)$ were incubated for 24 hours with the TLR9-agonist ODN2395, alone (-) or in combination with the IFN- inhibitor IFN alpha-IFNAR-IN-1 hydrochloride (+). MVs released into the culture supernatants were subsequently isolated by differential centrifugation, quantified and characterized with respect to expression of G3BP and DNA by flow cytometry. (A) Concentration in the culture supernatant of G3BP-expressing MVs, DNA-expressing MVs, and G3BP/DNA double-positive MVs. (B) Corresponding concentration of MVs expressing these markers when co-stimulating T-cells with anti-CD3 antibody. (C) The G3BP-derived median fluorescence intensity (MFI) of the G3BPexpressing MV population and (D) the DNA-derived MFI of the DNA-expressing MV population released from the ODN2395-treated PBMCs in presence (black columns) or absence (gray columns) of anti-CD3 antibody (OKT3). Columns and error bars represent median values and interquartile range. $\left({ }^{*}\right) \mathrm{P}<0.1 .{ }^{*} \mathrm{P}<0.05$. 
Background Microvesicles (MVs) expressing the type 1 interferon (IFN)-inducible protein galectin-3 binding protein (G3BP) may play a pathogenic role in systemic lupus erythematosus (SLE). Co-expression of DNA on such MVs may render them immunogenic and target for anti-dsDNA antibodies. Little is known about the mechanisms underlying generation of this MV population. In this study, we investigated how Toll-like receptors, interferon- (IFN-) and $\mathrm{T}$ cells are related hereto in healthy subjects.

Methods Peripheral blood mononuclear cells (PBMCs) isolated from 12 healthy donors were stimulated in-vitro for 24 hours with a series of TLR-agonists or the T-cell activating antibody OKT3 or were subjected to apoptosis by incubation with staurosporine. MVs in the supernatants were subsequently isolated by differential centrifugation and were quantified and characterized with respect to expression of G3BP and DNA by flow cytometry.

Results Stimulation of PBMCs with the TLR9-agonist and strong IFN- inducer ODN2395 significantly increased the release of MVs expressing G3BP. A large proportion of these MVs expressed augmented levels of DNA on their surface. The production of MVs with this phenotype was markedly enhanced by co-stimulation of $\mathrm{T}$ cells. Furthermore, dependency on IFN- in the generation of G3BPexpressing MVs was indicated by a marked reduction following addition of the IFN- inhibitor IFN alpha-IFNAR-IN1 hydrochloride.

Conclusions The release of G3BP-expressing MVs from healthy donor PBMCs is induced by stimulation of TLR9 in an IFNdependent manner. The co-expression of DNA accessible for anti-DNA antibodies on these MVs may render them relevant in lupus pathogenesis.

Funding Source(s): Lundbeck Foundation, Rigshospitalets Fund for Research, Gigtforeningen

Effect of IFN- inhibition on TLR9-induced release of MVs from mononuclear cells.

\section{ZOSTER INFECTIONS INCREASE THE RISK OF FLARES IN PATIENTS WITH SYSTEMIC LUPUS ERYTHEMATOSUS}

${ }^{1}$ Fangfang Sun*, ${ }^{2}$ Shuang Ye. ${ }^{1}$ Ren Ji Hospital, South Campus, Shanghai Jiaotong University; '2Department of Rheumatology, South Campus, Ren Ji Hospital, School of Medicine, Shanghai Jiao Tong University

\subsection{6/lupus-2019-Ism.132}

Background The aim of this cohort study is to explore whether zoster infection could increase the risk of flares in patients with systemic lupus erythematosus.

Methods Patients who had zoster infection between January 2013 and April 2018 were included from the SLE database of Shanghai Ren Ji Hospital, South Campus. Patients with bacterial infections including urinary tract infection and bacterial pneumonia were selected as controls. Severe infections including fungi infections and septic shock were excluded. Besides, 200 SLE patients at the same time were set as general populations by systemic sampling. Baseline period was defined as three months before infections or admissions for general population, and risk period was within three months after infections or admissions. Characteristics and treatment strategies of baseline period including gender, age, disease duration, average daily dose of prednisone and immunosuppressive agents were collected. Flare was defined by SELENE SLEDAI criterion. Flare free

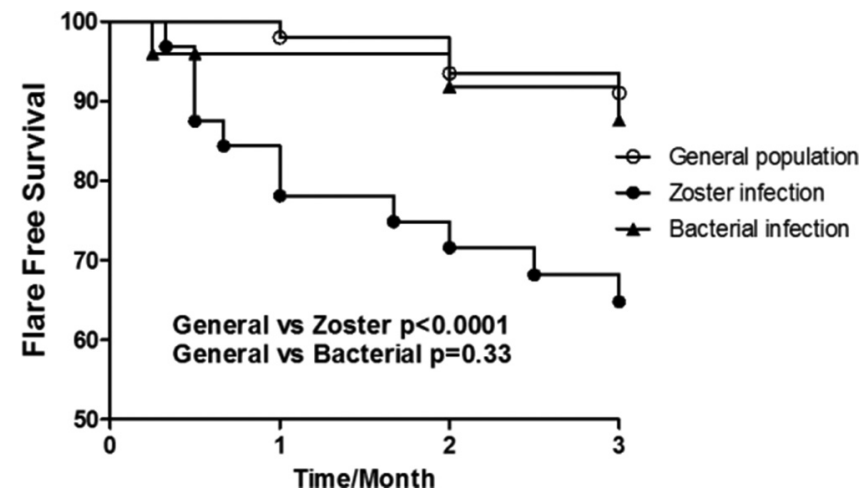

Abstract 132 Figure 1 Kaplan Meier curve showed that compared to general population, more patients with a history of zoster infectionwithin 3 months had a relapse of disease, while bacterial infections did not seem to affect the relapse rate

survival was assessed by Kaplan-Meier curve. Cox regression analysis was performed to identify risk factors of flares.

Results 33 with zoster infections, 18 with urinary tract infections and 7 patients with bacterial pneumonia were identified in this period. 13 relapses in 11 patients were observed, but there were only 3 relapses in bacterial infection group. Kaplan Meier curve showed that compared to general population, more patients with a recent history of zoster infection within 3 months had a flare $(33.3 \%$ vs $9 \%, \mathrm{p}=0.0034)$, while bacterial infections did not seem to affect the flare rate $(12 \%$ vs $9 \%, \mathrm{p}=0.4755)$. Furthermore, after adjusting important covariates, cox regression analysis confirmed that prior zoster infection within three months was a risk factor of flares in SLE patients $(\mathrm{p}<0.001$, HR 4.7).

Conclusions In patients with SLE, recent zoster infection within three months may be associated with increased risk of flares.

Funding Source(s): Kaplan Meier Curve of patients in three groups

\section{A YOUNG GIRL WITH LUPUS, RECURRENT PERICARDIAL EFFUSION AND CARDIAC TAMPONADE}

${ }^{1}$ Gummadi Anjani*, 'Rakesh Pilania, ${ }^{2}$ Anju Gupta, ${ }^{3}$ Deepti Suri, ${ }^{3}$ Surjit Singh. ${ }^{1}$ Dept. of Pediatrics, Allergy- Immunology Unit, Postgraduate Institute of Medical Education and Research; ${ }^{2}$ PGIMER Chandigarh; ${ }^{3}$ Postgraduate Institute of Medical Education and Research, Chandigarh, India

\subsection{6/lupus-2019--Ism.133}

Background Pleuritis and pericarditis,with or without effusion, are the commonest pulmonary and cardiovascular manifestations in children with systemic lupus erythematosus(SLE).However,SLE presenting as an isolated pericardial effusion with cardiac tamponade is distinctly unusual.

Methods We report one such case who went on to develop recurrent episodes of pericardial effusion and tamponade.

Results A-14-year-old girl presented with high grade fever, progressive dyspnea,pedal edema,periorbital puffiness for 7 days.She also had history of malar rash and photosensitivity. There was no history of oral ulcer and hair loss.Physical examination showed a prominent malar rash;heart rate 140/ min;respiratory-rate 40/min;muffled heart sounds; 